\title{
Invoking Behavioral Patterns on Autonomous Virtual Agents by Fuzzy Cognitive Maps and Video Games Techniques Using Modern Tools
}

\author{
J. Carlos Conde R., Abraham Sánchez L., and Abraham Sánchez F. \\ Benemérita Universidad Autónoma de Puebla, \\ Computer Science Department, Puebla, \\ Mexico \\ \{juanc.conde, asanchez\}@cs.buap.mx, axy72@hotmail.com
}

\begin{abstract}
In order to increase the game characters realism and its artificial environments, recent research has focused on improving the autonomous adaptation in real-time of characters to particular situations. This work resume an experimental process for creating Autonomous Virtual Agents (AVAs). Our work shows the main phases where an appropriate cognitive modeling leads to suitable responses from external stimuli and internal desires. As first proof of this, an undersea world is built and used to test the perception capabilities of artificial fish and sharks. This behavioral system is based on MoCAMG cognitive architecture following a methodology that combines common video-game techniques with non classical methods of AI. The proposal highlights how simple Fuzzy Cognitive Maps allows to get high-level control and generate simple behavioral patterns equivalent to habits through realistic perception capabilities.
\end{abstract}

Keywords: Fuzzy cognitive maps, autonomous virtual agents, artificial life.

\section{Introduction}

According to Computer Graphics Modeling hierarchy considered in [8] by John Funge, Xiaoyuan Tu and Demetri Terzopoulos, to obtain realistic behaviors on Autonomous Virtual Agents (AVAs) the new apex is the Cognitive Modeling. Studies on living beings provide psychological, anatomical and ethological information, so many works argue that an appropriate cognitive model could provide enough information to generate realistic simulations of artificial life and validate these by theoretical models. Actually, video-games and auto-animations techniques facilitate realistic behaviors on characters.

This work focuses on the most important aspects for modeling AVAs in a synthesized way. It proposes to use a own architecture for cognitive modeling which combines Fuzzy Cognitive Maps (not augmented and not nested) and video-games techniques for blending animation and sensing. As result it obtains 
easily several behavioral patterns, without having to define every single detail of behaviors. In particular, it reproduces common habits of fish in an undersea world. Unlike other approaches, in this way the programmer do not need to know each and every one of possible world states using a complex data structure. Therefore, above is a proof of the high-level control obtained in this work.

The content of this paper is as follows. Section 2 describes what is the most important behavioral components in living beings. A key piece is knowledge representation which is addressed in Section 3. Use of Fuzzy Cognitive Maps for this approach is discussed in Section 4. Related work is presented in Section 5. As part of this proposal, Section 6 describes MoCAMG architecture for cognitive modeling and an overview of methodology of implementation. As proof MoCAMG, methodology and FCMs are used for implementing a simulation of artificial life using a set of fish and shark as predator in Section \%. Furthermore, this section shows the behavioral patterns obtained. Conclusions and future work are presented in Section 8 .

\section{Behavioral Elements}

Most important objectives in living beings are survive or reproduce, these are goals that can be decomposed into other more immediate. Animals have developed acute perception modalities to detect opportunities and threats in their habitat. They focus their attention in two ways: (1) using their specialized sense organs and (2) through cognitive attention. Therefore, the factors that must be considered by a behavioral system are [18]: the environment, external stimuli and desires, the action selection after obtaining sensory information and the behavioral animation which introduce motion control algorithms.

This situation lead to a design problem that can be solved by identifying the principles for which living beings select their actions, that is, the priorities for the different behaviors. An action selection mechanism based on reasoning involves the use of AI techniques and is called task-level motion planning. Meanwhile the behavior that enables artificial beings become autonomous and "survive" in dynamic environments is a primitive behavior: reactive or adaptive behavior $[13,17,14]$. According to Jafreezal Jaafar in [10], it should be considered specific elements for modeling an Intelligent Virtual Agent which is the basis of an AVA.

\section{Knowledge Representation}

Since the aim is to facilitate the inference of decisions, representation of information together with an interpretation theory give meaning to phrases of logic. In this case, an AVA should execute and action of "perception" or sensing to update its information about the world and then enable a re-planning process of actions. So is critical to select an appropriate architecture to implement effective cognitive models using a viable approach to represent uncertainty.

In most of related literature, precondition axioms define statements to specify what is the state of the world before performing an action. The outcomes of an 
action are represented by effect axioms. Actions, effect axioms and precondition axioms can be expressed as a tree data structure where nodes are all possible situations, effect axioms describe the characteristics of each situation (root corresponds to initial situation $s_{0}$ ) and precondition axioms allow to know which are the sequence of permitted actions, as seen in Figure 1. Each pathway on the tree represents one possible action sequence. Whether some situations (nodes) are defined as targets then is possible to use programming based in conventional algorithms for performing searches. The aim is to obtain one actions sequence which leads to character or AVA towards its objective [8].

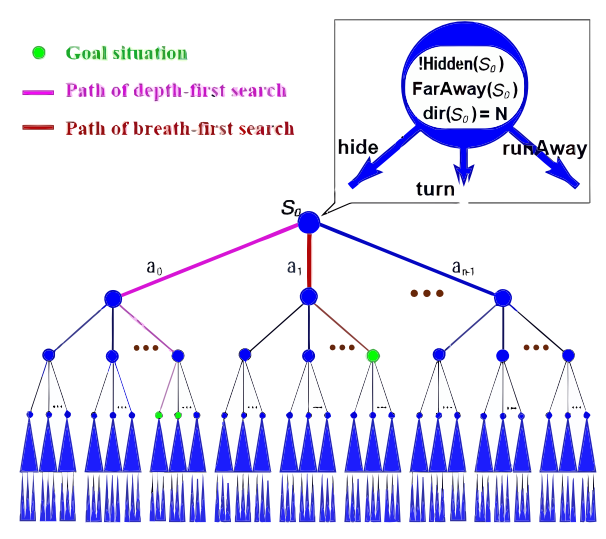

Fig. 1. Knowledge representation using "situation tree" presented in [8].

\section{Fuzzy Cognitive Maps as a Viable Option}

Mathematical models such as Navier-Stokes equations, Inverse Kinematics equations or even coupled differential equations are models that help to represent changes in virtual worlds. The main disadvantage is that these are hard to find, hard to solve and hard to run in real-time. Usually situation trees "facilitate" action selection process updating the stored knowledge. However, each inference uses only a small part of stored knowledge, and even the programer have to anticipate exactly what action could be selected in all possible conditions.

In contrast, Fuzzy Cognitive Maps (FCMs) [11] are a viable way to represent feedback because these are causal networks represented by fuzzy digraphs (see Figure 2), where nodes stand for concepts, actions or desires, and causal edges state the fuzzy rules between nodes. Furthermore, each input fires all the rules to some degree for modeling the "circular causality" of realistic virtual worlds.

FCMs by themselves act like a nonlinear dynamic system (such as an ANN) wherein all inputs are mapped to equilibrium states as output. Thus, a pathway in a simple FCM ends at a fixed point or in a finite loop. 


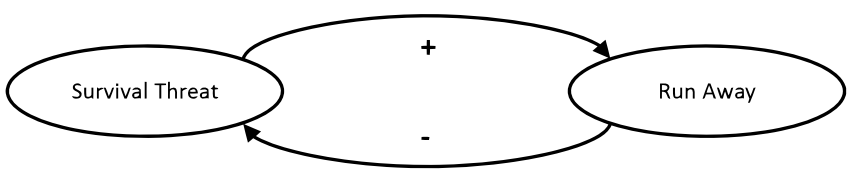

Fig. 2. Changes in a virtual world are causal and at the same time this causality feedback others [6].

\section{Related Work}

All the discussions point to the position that computational cognitive models can be true theories of cognition. Peter C. Pantelis and his team says in [15] that behavior of AVAs are modulated by a small number of distinct "mental states": exploring, gathering food, attacking, and fleeing. In two experiments, they studied subjects' ability to detect and classify the agents' continually changing mental states on the basis of their motions and interactions. The data provide intriguing evidence about the factors that influence over mental state, a key step towards a true "psychophysical intention".

Moreover, to get a believable behavior in characters the challenges are laid on generating on the fly flexible motion and complex behaviors inside of environments using realistic perception capabilities. N. Magnenat-Thalmann and D. Thalmann present in [12] research results which highlights interactivity and group behaviors are important parameters to test a cognitive model since realistic perception of environments and internal estate of the "actor" can be reflected affecting its behavior. Other interesting work more nearby to our approach is proposed by Toni Conde in [4] who presents two novel methodologies: the first technique integrates persistency to obtain a cognitive map of the perceived, the second technique integrates a perception approach by including the faculty of prediction, e. g., the orientation of the AVA attention.

Nevertheless, there are few works about Cognitive Architectures to improve AVAs in 3D environments using modern tools. A close architecture to this area is the RASCALS cognitive architecture presented in [2] which has an high expressive power to building advanced synthetic characters. But this is focused only on human cognition and it use an approach of Natural Language Generation (NLG) for the communication user-character.

In contrast, this work focus on cognitive modeling of autonomous agents or characters (not only humans) through endowing them with a certain amount of directives and letting them to generate the details by themselves. This approach considers the importance of modeling primitive actions properly and integrate them into complex actions combining an architecture, FCM and video-games techniques for blending animations and sensing. The component which is responsible of evoking "habits" considers the use of simple Fuzzy Cognitive Maps for modeling motivations in AVAs, but this could change using another approach of IA. 


\section{MoCAMG Architecture for Cognitive Modeling}

The Movis' Cognitive Architecture for Modeling Game-characters (MoCAMG, Fig. 3) is a hybrid architecture based on the well known cognitive architectures ACT-R and Soar. This provides a conceptual frame work to create models of how performing tasks that involve movement. As ACT-R and Soar, MoCAMG has two types of memory; the declarative memory is associated with removable goals, so is regarded as a short-term memory. Procedural memory adds, updates but no removed "rules" in run-time as in a long-term memory.

A key component is the Intentions Generator because this allows to obtain complex behaviors considered as habits. This plays a role of behavior arbitrator that considers the internal and external state of a virtual agent.

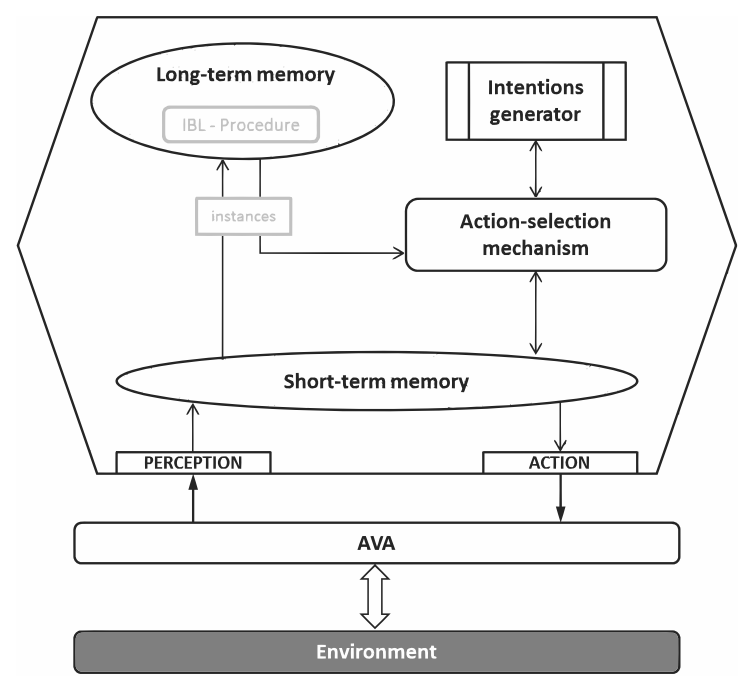

Fig. 3. MoCAMG architecture for cognitive modeling of Autonomous Virtual Agents.

Finally, we are currently working in the $I B L$-Procedure which serves to select an appropriate response (action) from a set of instances defined by situations (internal and external state) stored in the long-term memory. The purpose of this component is to obtain more weighted responses. However, the scope of this work focuses on the basis of reactive behavior to achieve integration and functionality in a simple way; using simple FCMs. Therefore, Intentions Generator can evoke "habits" or behavioral patterns through a high-level control (without defining every detail of movement). This is an important step towards more realistic behaviors. 


\subsection{Component-Based Behavioral System}

As overview of the methodology used and to synthesize the cognitive modeling process in AVAs, a diagram of workflow implemented is shown in Fig. 5. This is based on the hierarchy of behavioral animation modeling since it has made impressive strides toward autonomy resulting in virtual characters auto-animated endowed of more realistic behaviors $[9,8,7,5]$.

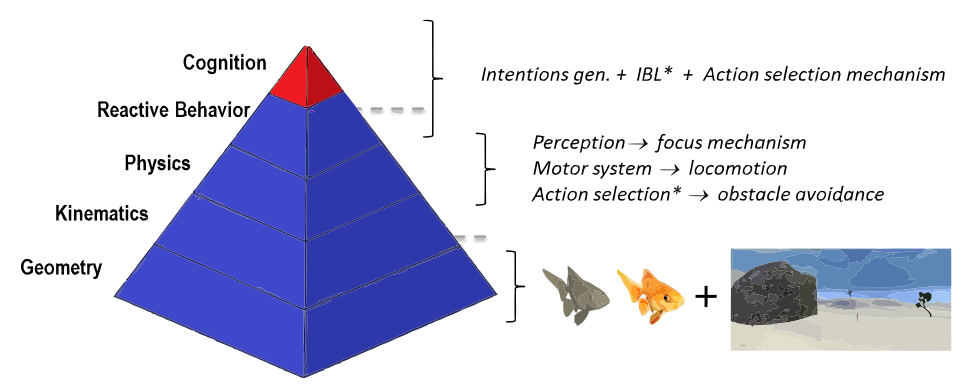

Fig. 4. Computer Graphics Modeling (CGM) hierarchy.

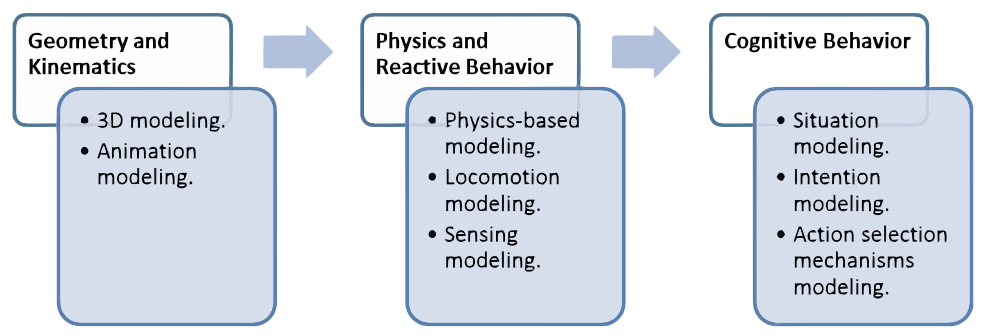

Fig. 5. Workflow for cognitive modeling which considers three levels of modeling: realistic appearance, realistic smooth and flexible motion and realistic high-level behaviors.

At this point is important to specify that from now on it will consider a desire is a potential influence of intention. An agent may have multiple desires, but only those which are the strongest desires will be considered collectively as intention. Additional information should be represented as qualitative recommendations of actions (referred as motor preferences) then is feasible to implement an Intention Generator using non-classical techniques of AI. 
Invoking Behavioral Patterns on Autonomous Virtual Agents by Fuzzy Cognitive Maps and ...

\section{$7 \quad$ Implementation of Autonomous Virtual Fish Using MoCAMG Architecture}

Fish and undersea world were implemented to test complex movements in full 3D space and to simulate the paradigm of artificial life. This consist of several goldfish, a shark, plants and different obstacles. This simulation was developed programming in LUA and using ShiVa3D as game engine.
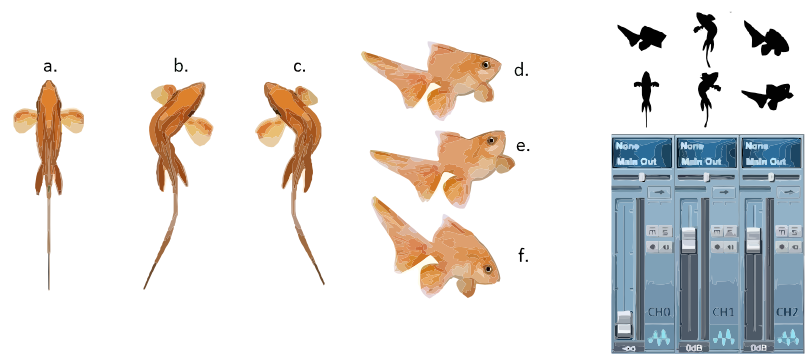

Fig. 6. Left: atomic movements. Right: manager of animations scheme.

The anatomy and movement form are set according to the related ethology [16]. This was modeled as shown in Figure 6 (left), where the combination of atomic actions generates necessary movement sequences. This represents the main idea of Barthel, Dannenmann and Hagen [1] where behaviors can be either low-level or high-level. Two or more simple movements can be executed simultaneously, as long as these are non-exclusive movements, through a component named manager of animations (scheme on the right side in Figure 6).

The implemented perception system considers four sensing models: (1) Avoidance of collision with terrain, (2) Avoidance of collision with fixed obstacles, (3) Avoidance of collision with dynamic obstacles and (4) Physical touch.

Capability to avoid collision with terrain was designed to be activated only when the agent is displacing using a ray casting method, it draws a distinction between go forward and go backward. Avoidance of collision with fixed obstacles is less simple; real fish use landmarks to generate mental maps of geometric relationships [3], so the calculation of attractive potential field is viable way to define a target in the space while calculation of repulsive potential field serves to avoid "known" obstacles.

However, fish possess highly developed sense organs, like chemoreceptors which detect gentle currents and vibrations (e. g. lateral line system). Therefore, to model similar sensory capabilities this implementation models the extended perceptual range as spherical sensor that detects other similar sensors. A visual perception model and the extended perception model together can detecting moving objects or even other cognitive entities (see Figure 7). Subsequently, is necessary to simulate physical contact to define more complex behaviors. So 
a ragdoll is used to simulate catching of food and physical touching in group behaviors.

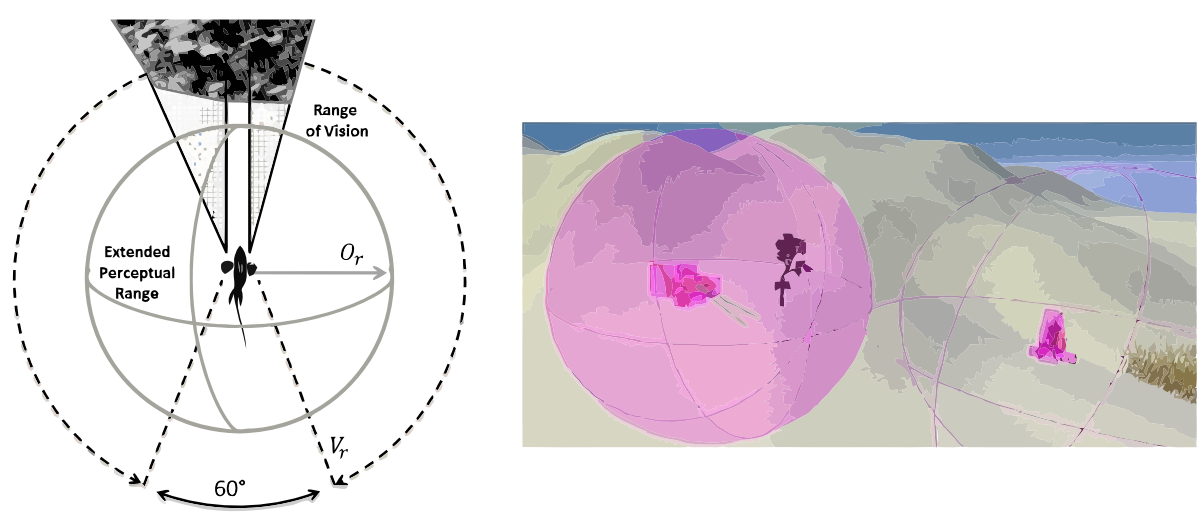

Fig. 7. Sensing modeling which attempts to simulate special skills of perception in fish (range of vision $V_{r}$ and extended perceptual range $O_{r}$ ).

Updating process of cognitive agent's world model is also a primitive action. In turn, this primitive action requires sensing actions where the behavioral system selects which to execute to discard unnecessary sensory information. The reactive system is responsible for executing motor controls efficiently, but this works with the action selection mechanisms to manage the suitable perception model and the most useful motor controller. Intentions generator controls these subsystems in a higher level to satisfy imperative desires at a given moment.

A hierarchy of intentions considered in [18], establishes theoretical basis to define the following scheme for this implementation.

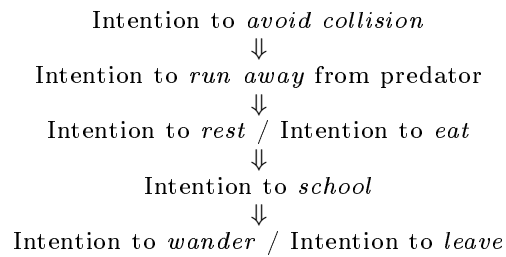

Intention to avoid collision is directly related with the data obtained from the sensing system, i. e., this is a reactive behavior. Intentions to wander and leave are the result of absence of other. However, for the implementation there are variables considered as desires; hunger, fatigue or survival threat, which governing in a sub-cognitive level all above intentions.

The FCM for a fish was taken from Julie A. Dickerson and Bart Kosko's work in [6] and this is shown in Figure 8. This is represented through a square matrix and each component takes values between -1 and 1 . Therefore the matrix establishes the causality flow between concepts of a virtual fish's FCM. 

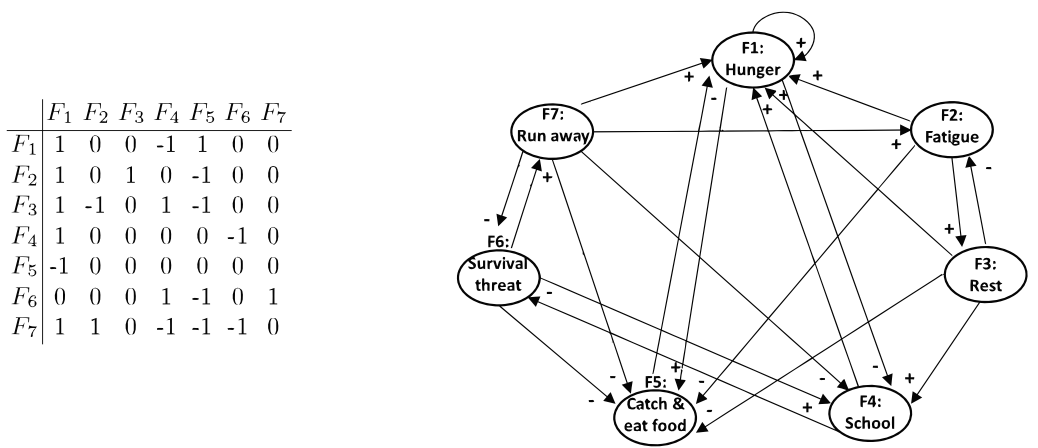

Fig. 8. Trivalent FCM implemented by Intentions Generator of virtual fish.

The FCM implemented for modeling an Intention Generator of a shark is based also in [6]. However, according to theory underlying, it was modified to obtain effective attracting points for the action selection mechanism. In this case, the action selection mechanism works as activation function for an ANN. Figure 9 shows the proposed FCM and its square matrix.
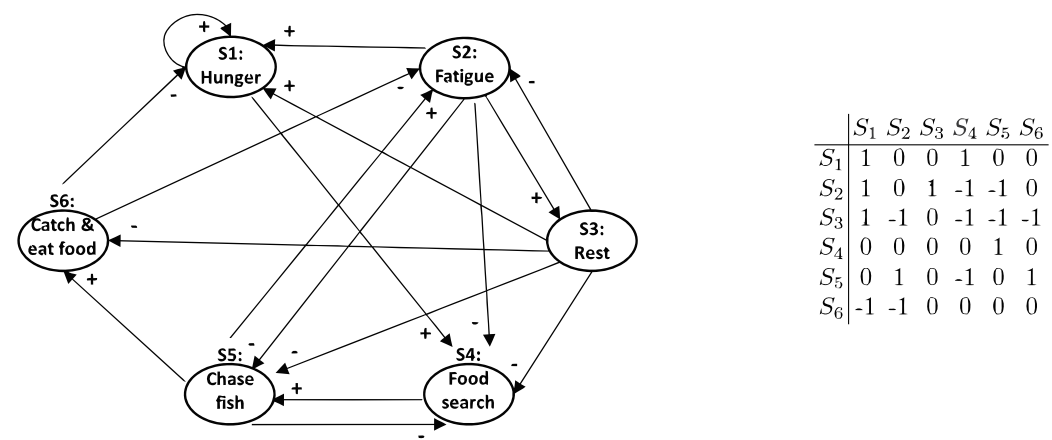

Fig. 9. Trivalent FCM implemented by Intentions Generator of virtual shark.

The approach presented in [6] proposes to use an augmented FCM to implement a virtual undersea world in $2 \mathrm{D}$, where causal links are all listed in a single square matrix. However, the approach of this work is more ambitious because it attempts to test all cognitive capabilities defined by the proposed MoCAMG architecture inside of a 3D environment. Even the approach of Julie A. Dickerson and Bart Kosko considers an explicit "mental" connection between characters, which is impossible in real world, at least so far.

Considering all above, the contribution is resumed in the Algorithm 1. This represents the integration of several approaches in this area. Proposed algorithm could be generalized to other characters, specially for those with similar cognitive 
capabilities. This proves the usability of the architecture and FCMs.

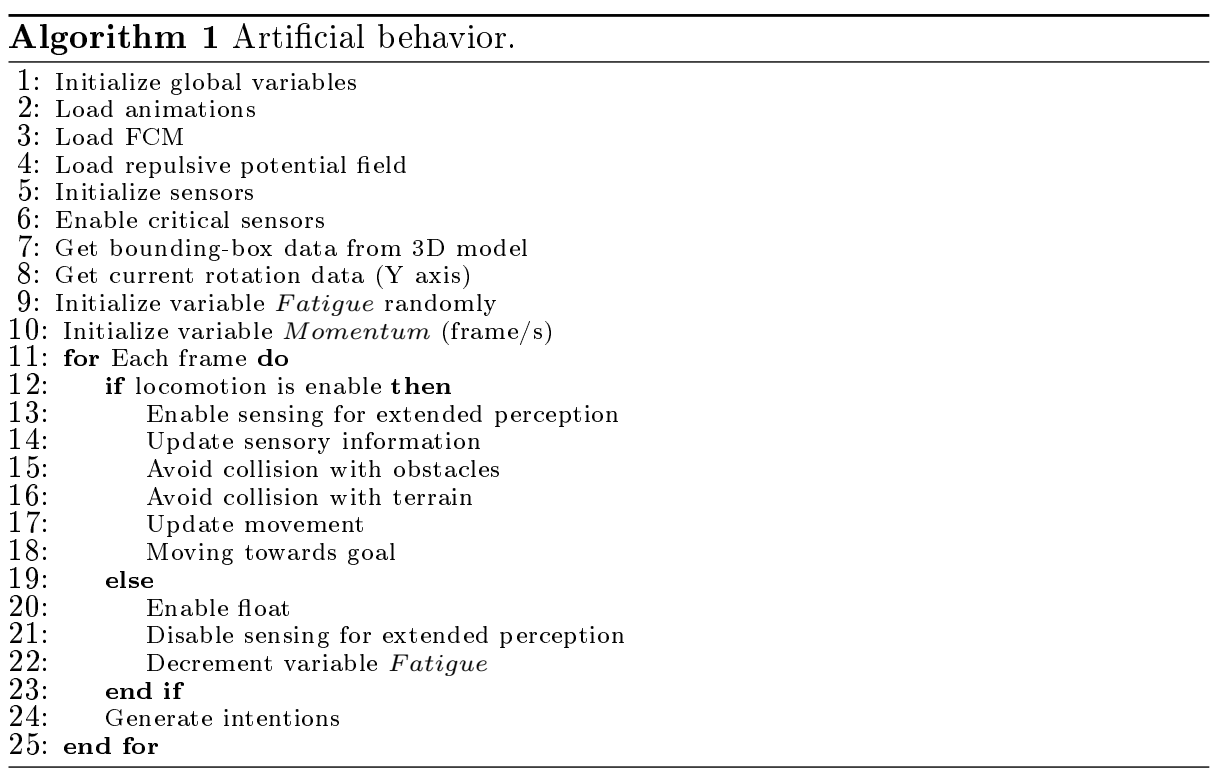

\subsection{Resulting Behavioral Patterns Using FCMs}

Resulting information from the sensing system and internal state enables Intentions Generator to fire the current state into the FCM. This is represented by a vector of seven components: $C_{i}(t)=\left[F_{1} F_{2} F_{3} F_{4} F_{5} F_{6} F_{7}\right]$. Bounded loops form hidden behavioral patterns through nested loops observed in Figure 10.
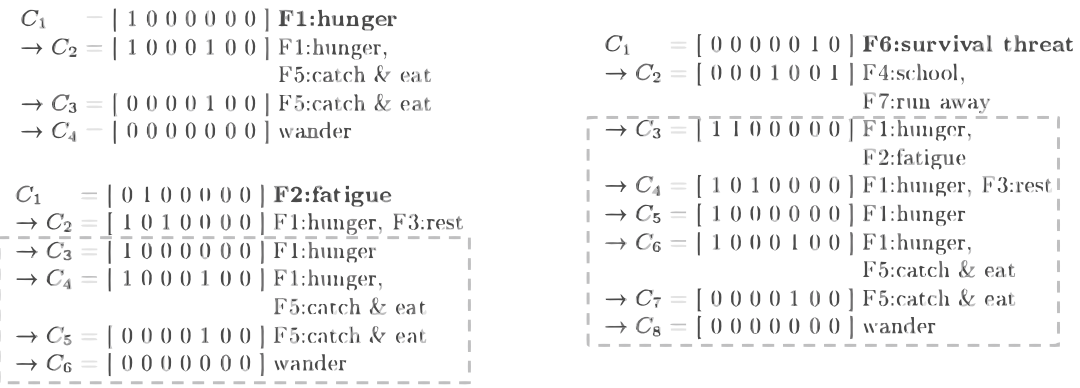

Fig. 10. Behavioral patterns for virtual fish determined by bounded loops. 
Invoking Behavioral Patterns on Autonomous Virtual Agents by Fuzzy Cognitive Maps and ...

Since behavioral patterns can be considered as habits, this are described in Figure 10. Notice that in several cases there are more than one component set in one, so note the importance of implement a hierarchy of intentions into the Action Selection Mechanism.

As proof of the simple way to cognitive modeling using MoCAMG, FCMs and a game engine, a predator character was added using basically the same algorithm and changing the data structure of its FCMs. According to ethology, the artificial predator is a black-tips shark. In this moment the character has an acceptable reactive behavior.

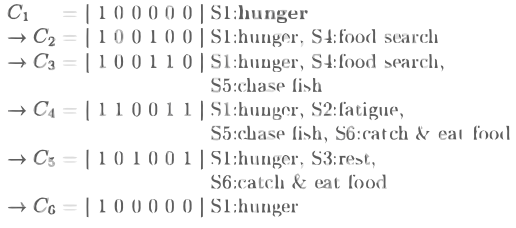

Fig. 11. Behavioral patterns for virtual shark determined by permanent desires of hunger or fatigue.

\section{Conclusions and Future Work}

This work represents a very important step towards the generation of cognitive characters with realistic behaviors, in a synthesized way. This is an important approach to obtain autonomous characters or AVAs combining video-games techniques. The proposal highlights how behavior organized in hierarchical way leads to obtain a high-level control which compete for reflexes rather than reflexes compete for control of "muscles" or other details. Even, this first implementation serves as an useful tool to test and improving other cognitive procedures.

There is a lot of future work, which is good because this attempts to explore more cognitive phenomena related with long-term memory. One of these is the Instance Based Learning based on the Instance Based Theory. And regarding the tests, it will be necessary to introduce more predator characters and cause antagonism defining another role, specifically introducing a dolphin character. But prior this work should must evolve to improve cognitive capabilities to evoke more complex behaviors, thus generating artificial learning and reasoning.

\section{References}

1. Barthel, H., Dannenmann, P., Hagen, H.: Towards a General Framework for Animating Cognitive Characters. In: Proceedings of the 3rd IASTED International 
Conference on Visualization, Imaging, and Image Processing. VIIP'03, Benalmádena, Spain (2003)

2. Bringsjord, S., Khemlani, S., Arkoudas, K., Mcevoy, C., Destefano, M., Daigle, M.: Advanced synthetic characters, evil. In: and E," in Game-On 2005, 6th International Conference on Intelligent Games and Simulation (M. Al-Akaidi and. pp. 31-39. European Simulation Society (2005)

3. Chung, S.: Appropriate maze methodology to study learning in fish. University of Toronto Journal of Undergraduate Life Sciences 2(1) (2009)

4. Conde, T., Thalmann, D.: An integrated perception for autonomous virtual agents: active and predictive perception. Journal of Visualization and Computer Animation 17(3-4), 457-468 (2006)

5. Dannenmann, P., Barthel, H., Hagen, H.: Multi level Control of Cognitive Characters in Virtual Environments. In: Proceedings of the 14th IEEE Visualization 2003 (VIS'03). pp. 92-. VIS '03, IEEE Computer Society, Washington, DC, USA (2003)

6. Dickerson, J.A., Kosko, B.: Virtual Worlds as Fuzzy Cognitive Maps. Presence 3(2), 173-189 (1994)

7. Funge, J.: Cognitive modeling for games and animation. Commun. ACM 43(7), 40-48 (Jul 2000)

8. Funge, J., Tu, X., Terzopoulos, D.: Cognitive modeling: knowledge, reasoning and planning for intelligent characters. In: Proceedings of the 26th annual conference on Computer graphics and interactive techniques. pp. 29-38. SIGGRAPH '99, ACM Press/Addison-Wesley Publishing Co., New York, USA (1999)

9. Funge, J.D.: Making them behave: cognitive models for computer animation. Ph.D. thesis, University of Toronto, Toronto, Ont., Canada (1998)

10. Jaafar, J., McKenzie, E.: A reactive architecture for autonomous agent navigation using fuzzy logic. The University of Edinburgh, Edinburgh, UK (2009)

11. Kosko, B.: Fuzzy cognitive maps. International Journal of Man-Machine Studies (24), 65-75 (1986)

12. Magnenat-Thalmann, N., Thalmann, D.: Virtual humans: thirty years of research, what next? The Visual Computer 21(12), 997-1015 (2005)

13. Manning, A., Dawkins, M.: An Introduction to Animal Behaviour. Cambridge University Press (1998)

14. McFarland, D.: Animal behaviour: psychobiology, ethology, and evolution. Longman Scientific and Technical (1993)

15. Pantelis, P.C., Cholewiak, S., Ringstad, P., Sanik, K., Weinstein, A., Wu, C.C., Feldman, J.: Perception of intentions and mental states in autonomous virtual agents. Cognitive Sciences Society 33rd Annual Meeting pp. 1990-1995 (2011)

16. Stephens, K., Pham, B., Wardhani, A.: Modelling fish behaviour. In: Proceedings of the 1st International Conference on Computer Graphics and Interactive Techniques. pp. 71-78. GRAPHITE '03, ACM, New York, USA (2003)

17. Tinbergen, N.: The study of instinct. Clarendon Press (1951)

18. Tu, X.: Artificial animals for computer animation: biomechanics, locomotion, perception, and behavior. Springer-Verlag, Berlin, Heidelberg (1999) 